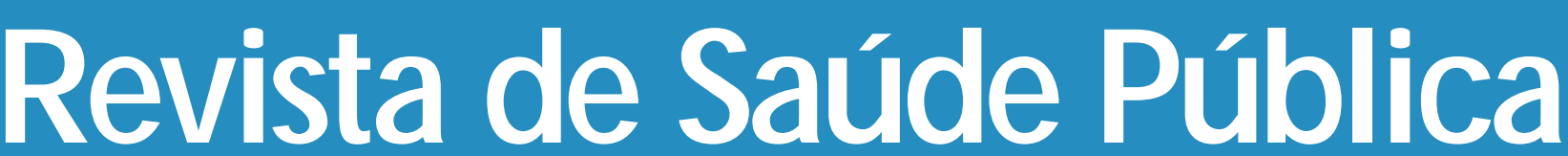

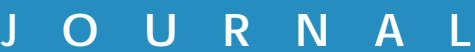

0 F

$\mathbf{P} \mathbf{U}$

B L I C

H E A L T

\title{
Valor preditivo do "Clinical Risk Index for Babies" para o risco de mortalidade neonatal
}

\author{
Predictive value of the "Clinical Risk Index for Babies" \\ for the risk of neonatal death
}

O scar T. Matsuoka, Lilian S.R.Sadeck, Jesselina F.S. H aber, Renata S.M. Proença, Marta M.G.Mataloun, José L.A. Ramos e Cléa R. Leone

Instituto da Criança "Prof. Pedro de Alcântara" do Hospital das Clínicas da Faculdade de Medicina da U niversidade de São Paulo (FMU SP). São Paulo, SP - Brasil (O.T.M., L.S.R.S., R.S.M.P., M.G.M., C.R.L.), Departamento de Pediatria da FMU SP. São Paulo, SP - Brasil (J.L.A.R.)

MATSU O KA O scar T. , Lilian S.R.Sadeck, Jesselina F.S. H aber, Renata S.M. Proença, Marta M.G .Mataloun, José L.A. Ramos e Cléa R. Leone Valor preditivo do "Clinical Risk Index for Babies" para o risco de mortalidade neonatal Rev. Saúde Pública, 32 (6): 550-5, 1998

(c) Copyright Faculdade de Saúde Pública da USP. Proibida a reprodução mesmo que parcial sem a devida autorização do Editor Cient́fico. Proibida a utilização de matérias para fins comerciais. All rights reserved. 


\title{
Valor preditivo do "Clinical Risk Index for Babies" para o risco de mortalidade neonatal
}

\author{
Predictive value of the "Clinical Risk Index for Babies" \\ for the risk of neonatal death
}

\author{
O scar T. Matsuoka, Lilian S.R.Sadeck, Jesselina F.S. H aber, Renata S.M. Proença, Marta \\ M.G.Mataloun, José L.A. Ramos e Cléa R. Leone \\ Instituto da Criança "Prof. Pedro de Alcântara" do Hospital das Clínicas da Faculdade de Medicina \\ da Universidade de São Paulo (FMUSP). São Paulo, SP - Brasil (O.T.M., L.S.R.S., R.S.M.P., M.G.M., \\ C.R.L.), Departamento de Pediatria da FMUSP. São Paulo, SP - Brasil (J.L.A.R.)
}

\begin{abstract}
Resumo
Objetivo Comparar o valor preditivo do CRIB (Clinical Risk Index for Babies) para o risco de mortalidade neonatal ao peso de nascimento (PN) e idade gestacional (IG).

Método Numa coorte prospectiva foram estudados, durante o ano de 1996, 71 recémnascidos admitidos na unidade de terapia intensiva, com PN $<1.500 \mathrm{~g}$ e/ou IG $<31$ semanas.

Resultados A taxa de mortalidade foi de $29,6 \%$; para PN $<1.000 \mathrm{~g}$ ou IG $<29$ semanas foi de $60 \%$, enquanto que para o escore de CRIB $>10$ foi de $100 \%$.

Conclusões O escore de CRIB > 10 correspondeu a maiores especificidade e valor preditivo positivo em relação aos demais parâmetros. A área determinada pela "receiver operating characteristic" relativa ao CRIB também foi superior. O CRIB mostrou-se um marcador mais acurado na previsão de risco de mortalidade quando comparado ao PN ou IG isoladamente.

Mortalidade neonatal [Saúde pública]. Peso ao nascer. Idade gestacional. Unidades de terapia intensiva neonatal.
Abstract
Objective
Several indicators, mainly birthweight and gestational age, have been used to predict the mortality risk in neonatal intensive care units. In order to assess the potential value of CRIB in predicting neonatal mortality, the score was used over the first 12 hours of life of the newborns admitted to this unit, during the year of 1996.

Method The inclusion criteria consisted of all infants without inevitably lethal congenital malformations, birthweight below 1,500g and/or gestational age less than 31 weeks. Newborn children who died within 12 hours after delivery were excluded. The CRIB score covers birth weight, gestational age, the presence of congenital malformations (not inevitably lethal) and three indexes of physiological status during first 12 hours after birth - maximum and minimum appropriate fraction of inspired oxygen and maximum (most acidotic) base excess.
\end{abstract}

Correspondência para/Correspondence to: Oscar Tadashi Matsuoka - Rua João Ramalho, 324/82 - Perdizes - 05008-001 São Paulo, SP - Brasil. E-mail: Edição subvencionada pela FAPESP (Processo n 97/09815-2).

Recebido em 3.11.1997. Reapresentado em 3.4.1998. Aprovado em 6.5.1998. 
Results In a prospective cohort, seventy one newborn children were studied. The birthweight (average) was $1,119 \pm 275.6 \mathrm{~g}$, gestational age 30 weeks $4 / 7 \pm 2$ weeks 3/7; male (57\%); Apgar $1^{\circ}$ min. score $\leq 3$ (36.2\%) and Apgar $5^{\circ} \mathrm{min}$. score $<5(5.8 \%)$. The mortality rate was $29.6 \%$ (gold standard). But mortality rate by birthweight less than 1,000 gr. or gestational age lower than 29 weeks was $60.0 \%$ and for the CRIB score above 10 was $100 \%$.

Discussion

\begin{abstract}
The specificity and predictive positive values for CRIB score above 10 were greater than any other two parameters. The area under the receiver operating characteristic (ROC) curve for predicting death was significantly greater for CRIB than for birthweight alone. It was concluded that the $C R I B$ score is a better predictive indicator for mortality than are birthweight and gestational age.
\end{abstract}

Neonatal mortality [Public health]. Birth weight. Gestacional age. Intensive care units, neonatal.

\section{INTRO DU ÇÃO}

A variação da taxa de mortalidade que existe entre diferentes serviços neonatais constitui tema muito discutido na literatura ${ }^{2,3,4,9,12,14}$. Atualmente sabe-se que esta diferença não pode ser interpretada apenas como conseqüência da qualidade da assistência médica prestada. Isto porque, como parâmetro isolado, a taxa de mortalidade não pondera as condições clínicas na ocasião da admissão no serviço. Na área neonatal, essas diferenças sempre foram corrigidas analisandose o peso de nascimento e a idade gestacional. Porém, variações residuais ainda ficavam evidentes, provavelmente em decorrência de outros fatores, tais como a gravidade de patologias respiratórias ou presença de malformações congênitas ${ }^{7}$.

A partir da década 80, desenvolveram-se índices para avaliar a condição fisiológica de pacientes criticamente enfermos admitidos em unidade de terapia intensiva (UTI), inicialmente na área de adultos ${ }^{8}$ e posteriormente na área pediátrica ${ }^{10}$ e neonatal ${ }^{11}$. Procurava-se definir a gravidade e instabilidade do paciente através de escores, constituindo-se em marcadores quantitativos do risco de mortalidade durante a internação hospitalar.

$\mathrm{O}$ "Clinical Risk Index for Babies" $(\mathrm{CRIB})^{13}$ foi desenvolvido em 1993 e se baseia no registro de dados rotineiramente pesquisados nas primeiras doze horas após o nascimento, consistindo num método simples e de fácil aplicação. O índice foi utilizado inicialmente para avaliar a qualidade da assistência médica intensiva, comparando-se a taxa de mortalidade entre nove hospitais terciários e quatro não terciários do Reino Unido. Não foram detectadas diferenças significantes nas taxas de mortalidade entre os dois grupos. No entanto, quando foi utilizado esse escore, observou-se que a chance de morte foi duas vezes maior nos hospitais não terciários.

Como consequiência desse estudo, o escore passou a ser valorizado como um possível marcador do desempenho de unidades neonatais, podendo vir a ser utilizado como um instrumento mais preciso na vigilância da qualidade da assistência médica, podendo inclusive seus resultados servirem de base para orientar diretrizes de políticas de saúde pública.

No Brasil, esse método começa a ser testado em alguns serviços neonatais. A divulgação desta experiência, realizada de uma forma controlada cientificamente, poderá contribuir para validar sua utilização em diferentes unidades, a fim de que a atuação de cada uma possa ser melhor avaliada.

Nesse contexto, desenvolveu-se estudo para avaliar a capacidade do escore do CRIB em prever a mortalidade de recém-nascidos $(\mathrm{RN})$ admitidos em UTI, comparando-se este escore a outros parâmetros normalmente utilizados para este fim, como peso de nascimento e idade gestacional.

\section{MÉTODO}

Realizou-se estudo prospectivo de coorte envolvendo todos os RN admitidos na UTI do berçário anexo à Maternidade de um hospital de clínicas de São Paulo, durante o período de 1 de janeiro a 31 de dezembro de 1996. Foram obedecidos os critérios de inclusão estabelecidos pelo "International Neonatal Network" ${ }^{13}$, ou seja: peso de nascimento $(\mathrm{PN})<1.500 \mathrm{~g}$ e/ou idade gestacional (IG) < 31 semanas. Foram excluídas do estudo as crianças que evoluíram para óbito com menos de doze horas de vida, bem como aquelas portadoras de malformações congênitas, inevitavelmente letais (anencefalia, trissomia do 18 e agenesia renal).

O escore do CRIB envolve análise das variáveis PN, IG, a presença de malformações congênitas (excluindo malformações inevitavelmente letais) e três índices do 
estado fisiológico: máxima e mínima fração inspiratória de oxigênio $\left(\mathrm{FiO}_{2}\right)$ utilizada e o valor mais elevado de excesso de bases (BE). Cada fator recebe pontuação que varia conforme a escala de gravidade, como demonstrado na Tabela 1.

\section{Delineamento da Pesquisa}

Uma vez admitido na UTI, cada RN foi acompanhado por uma equipe treinada para o preenchimento dos protocolos de pesquisa e que não interferiu sobre as condutas clínicas adotadas. Inicialmente foram registrados os dados maternos, condições de nascimento e o peso. Após o período de 12 horas de vida, foi aplicado o escore do CRIB, com base na evolução médica, tendo sido anotados os valores extremos da $\mathrm{FiO}_{2}$ que foram utilizados e o valor mais elevado do $\mathrm{BE}$ obtido pela gasometria arterial. $\mathrm{A} \mathrm{FiO}_{2}$ apropriada foi considerada aquela necessária para se obter uma $\mathrm{PaO}_{2}$ de 50 a $70 \mathrm{mmHg}$. Para as crianças que não receberam suporte respiratório e não necessitaram de coleta de gasometria, a $\mathrm{FiO}_{2}$ e $\mathrm{BE}$ foram considerados normais.

Tabela 1 - Construção do escore do CRIB.

\begin{tabular}{|c|c|}
\hline Fator & Pontuação \\
\hline \multicolumn{2}{|l|}{ Peso de nascimento $(\mathrm{g})$} \\
\hline$>1.350$ & 0 \\
\hline $851-1.350$ & 1 \\
\hline $701-850$ & 4 \\
\hline$\leq 700$ & 7 \\
\hline \multicolumn{2}{|l|}{ Idade gestacional (sem) } \\
\hline$>24$ & 0 \\
\hline$\leq 24$ & 1 \\
\hline \multicolumn{2}{|l|}{ Malformação congênita* } \\
\hline Ausente & 0 \\
\hline Sem risco iminente de vida & 1 \\
\hline Com risco iminente de vida & 3 \\
\hline \multicolumn{2}{|c|}{ Excesso de bases máximo nas $1^{\text {as }} 12 \mathrm{~h}$} \\
\hline$>-7,0$ & 0 \\
\hline$-7 a-9,9$ & 1 \\
\hline-10 a 14,9 & 2 \\
\hline$\leq-15,0$ & 3 \\
\hline \multicolumn{2}{|c|}{$\mathrm{FiO}_{2}$ mínima apropriada nas $1^{\text {as }} 12 \mathrm{~h}$ vivida } \\
\hline$<0,40$ & 0 \\
\hline $0,41-0,80$ & 2 \\
\hline $0,81-0,90$ & 3 \\
\hline $0,91-1,0$ & 4 \\
\hline \multicolumn{2}{|c|}{$\mathrm{FiO}_{2}$ máxima apropriada na $1^{\text {as }} 12 \mathrm{~h}$} \\
\hline$<0,40$ & 0 \\
\hline $0,41-0,80$ & 1 \\
\hline $0,81-0,90$ & 3 \\
\hline $0,91-1,0$ & 5 \\
\hline
\end{tabular}

*Excluindo malformações inevitavelmente letais.
A idade gestacional definitiva foi considerada da seguinte forma: (1) determinada pela data da última menstruação (DUM), quando confirmada pela ultrassonografia (USG), realizada antes da $20^{a}$ semana de gestação, ou (2) pelo método Dubowitz, quando a DUM diferia em 2 ou mais semanas em relação a USG ou a este método.

A observação de cada paciente foi realizada até a ocasião da alta hospitalar, com registro dos óbitos ocorridos.

A morte foi definida como óbito neonatal (até 28 dias), ocorrido durante a internação hospitalar.

\section{Análise Estatística}

Utilizou-se o programa Epi Info 6.0 para criação do banco de dados e a análise estatística foi realizada através do Statcalc.

Os dados populacionais tiveram análise descritiva apresentada em médias e desvio-padrão.

Foram calculados: sensibilidade, especificidade, valor preditivo positivo, valor preditivo negativo e risco relativo para escores de CRIB > 5 e $>10, \mathrm{PN}<750 \mathrm{~g} \mathrm{e}<$ $1.000 \mathrm{~g}$ e idade gestacional $<29$ semanas. Optou-se por estudar estes parâmetros porque na literatura são identificados como os de maior risco para morte neonatal.

Considerou-se a mortalidade real do período para cada um destes grupos como padrão de referência para o cálculo de significância estatística desses indicadores pelo método do Qui-quadrado ( $\mathrm{p}<0,05)$.

A análise da especificidade e sensibilidade do PN, IG e escore do CRIB para predição de morte for realizada graficamente através da curva "receiver operating characteristic" (ROC). A expressão quantitativa do poder de cada índice como marcador de previsão de morte foi avaliada através da área que cada curva determinou sobre o espaço delimitado pelo gráfico ${ }^{1,5}$.

\section{RESU LTAD O S}

Durante o período de estudo, 79 RN preencheram os critérios de inclusão. Deste total, 8 crianças foram excluídas ( 5 porque evoluíram para o óbito com menos de 12 horas de vida, duas foram encaminhadas para outro setor do complexo hospitalar e uma apresentava malformações incompatíveis com a vida).

O peso médio de nascimento foi de $1.119 \pm 275,6$ g e a idade gestacional de 30 sem e 4/7 \pm 2 sem e 3/ 7. Quarenta e um RN (57\%) eram do sexo masculino e $46(64,8 \%)$ eram brancos. Vinte e cinco RN $(36,2 \%)$ apresentaram Apgar de $1^{0}$ minuto $\leq 3$ e 4 crianças $(5,8 \%)$ apresentaram Apgar de $5^{0} \mathrm{~min} \leq 5$. $\mathrm{O}$ parto foi cesariana em $81,4 \%$ dos casos. Vinte e um RN (29,6\%) evoluíram para óbito.

A análise da mortalidade em relação ao CRIB, 
PN e idade gestacional encontra-se na Tabela 2.

Foram calculados a sensibilidade, especificidade, risco relativo e valor preditivo positivo e negativo segundo o $\mathrm{PN}<750 \mathrm{~g} \mathrm{e}<1.000 \mathrm{~g}$, idade gestacional $<29$ semanas e CRIB $<5$ e $<10$ ( Tabela 3).

A análise do comportamento do PN, IG e escore do CRIB quanto à especificidade e sensibilidade foi realizada graficamente através da curva ROC (Figura 1). A expressão quantitativa do poder de cada índice como marcador de previsão de morte foi avaliada através da área que cada curva determinou sobre o espaço delimitado pelo gráfico. A área determinada por cada curva foi progressivamente representada pela IG $(0,77), \mathrm{PN}(0,83)$ e escore do CRIB $(0,90)$, demonstrando a superioridade do último.

\section{DISCUSSÃO}

O escore do CRIB constitui passo importante na avaliação do desempenho de unidades neonatais. Além de valorizar o peso de nascimento e a idade gestacional, o método considera também a condição

Tabela 2 - Mortalidade neonatal segundo o peso de nascimento, idade gestacional e "CRIB".

\begin{tabular}{lcc}
\hline Variável & $\mathrm{N}$ & $\begin{array}{c}\text { Mortalidade } \\
\text { neonatal (\%) }\end{array}$ \\
\hline Peso ao nascer $\mathbf{( g )}$ & 12 & $66,6^{*}$ \\
$<750$ & 11 & $54,5^{*}$ \\
$750-999$ & 20 & 32,0 \\
$1.000-1.249$ & 28 & 4,7 \\
$1.250-1.499$ & & \\
Idade gestacional & 20 & $60,0^{*}$ \\
$23-28$ & 37 & 18,9 \\
$29-32$ & 14 & 14,2 \\
$33-36$ & & \\
CRIB & 45 & 2,2 \\
$0-5$ & 17 & $64,7^{*}$ \\
$6-10$ & 9 & $100,0^{*}$ \\
$11-15$ & 0 & - \\
$>16$ & 71 & $21(29,6)$ \\
\hline Total & & \\
\hline $\mathrm{N}=$ número de recém-nascidos & & \\
${ }_{p}<0,05$ & &
\end{tabular}

clínica do RN à admissão no serviço. Neste sentido, o escore associa à gravidade do paciente fatores demográficos normalmente utilizados como marcadores de mortalidade, constituindo-se num índice mais completo de avaliação ${ }^{6}$.

A mortalidade geral para a população estudada, durante o período, foi de $29,6 \%$. Entretanto, observa-se um comportamento heterogêneo de distribuição desta taxa, quando analisada sob o ponto de vista de estratificação do peso de nascimento, idade gestacional e CRIB. Nesse contexto, a mortalidade apresentou uma tendência a se concentrar nas faixas extremas de peso ( $\mathrm{PN}<1.000 \mathrm{~g}$ ), idade gestacional (IG < 29 semanas) e escore do CRIB (CRIB > 10).

De forma geral, o PN e IG sempre foram utilizados como parâmetros preditivos de mortalidade neonatal. A análise estatística dos resultados obtidos no presente trabalho também revelou esta correlação, que foi observada através do cálculo do risco

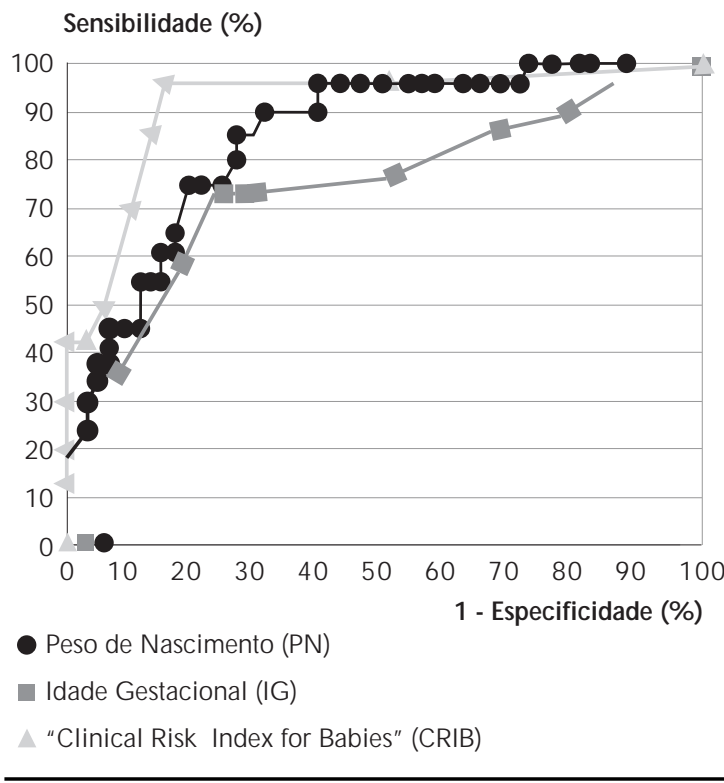

Figura - Análise do PN, IG , CRIB quanto à sensibilidade e especificidade através da curva "Receiver operating characteristic".

Tabela 3 - Avaliação do desempenho de diferentes indicadores preditivos da mortalidade neonatal.

\begin{tabular}{lccccc} 
Atributos & CRIB $>5$ & CRIB $>10$ & PN $<750$ & PN $<1.000$ & IG $<29$ \\
Sensibilidade (\%) & 90,9 & 40,9 & 36,4 & 63,6 & 54,5 \\
Especificidade (\%) & 88,0 & 100 & 92,0 & 82,0 & 84,0 \\
Valor preditivo negativo (\%) & 95,7 & 79,4 & 76,7 & 83,7 & 80,8 \\
Valor preditivo positivo (\%) & 76,9 & 100 & 66,7 & 60,9 & 60,0 \\
Risco relativo & 17,69 & 4,85 & 2,86 & 3,73 & 3,12 \\
Intervalo de confiança 95\% & $2,99-69,75$ & $2,99-7,87$ & $1,55-5,25$ & $1,83-7,61$ & $1,61-6,05$ \\
\hline
\end{tabular}

PN = peso de nascimento, IG = idade gestacional, CRIB = "Clinical Risk Index for Babies". 
relativo e intervalo de confiança. Neste contexto, os marcadores estudados não apresentaram diferença importante entre si e em relação ao escore do CRIB ( exceto para o escore $>5$, cujo intervalo de confiança de grande amplitude diminuiu o seu poder estatístico). A diferenciação do escore do CRIB mostrouse mais evidente na análise do seu valor preditivo positivo e negativo e principalmente na avaliação da relação entre especificidade e sensibilidade para predição de morte. Esta última relação pode ser expressa graficamente através da curva ROC, que é uitilizada para descrever a acurácia de um espectro de pontos de cortes.

A Figura 1 apresenta a curva ROC que foi construída para a análise do comportamento do PN, IG e escore do CRIB. Pode-se observar que o escore do CRIB se distribui no canto superior esquerdo do gráfico. Isto significa que na medida em que a sensibili-dade diminui, existe pouca perda da especificidade, caracterizando-se como um teste de bom poder discriminatório. Por outro lado, as curvas da IG e PN estão distribuídas mais próximas à diagonal. Nesta situação, na medida em que a sensibilidade diminui, ocorre grande variação na especificidade, demonstrando menor poder discriminatório dos testes.

A expressão quantitativa do poder de cada índice como marcador de previsão de morte pode ser avaliada através da área que cada curva determina sobre o espaço delimitado pelo gráfico. Neste sentido, o escore do CRIB demonstrou a sua superioridade com área de 0,90 . Por outro lado, o peso de nascimento determinou a área de 0,83 , resultado limitado que, entretanto, merece consideração principalmente pela praticidade de seu uso diário.
Particularmente, o escore do CRIB > 10 mostrouse mais eficiente na capacidade de previsão do risco de morte neonatal para a realidade da instituição analisada. Ele apresentou especificidade e valor preditivo positivo de $100 \%$, associado a um risco relativo de 4,85 e intervalo de confiança variando de 2,99-7,87. Desta forma, esta faixa do escore do CRIB compreende o grupo populacional no qual devem ser desenvolvidos estudos para redução da mortalidade. No estudo multicêntrico, desenvolvido no Reino Unido pelo "International Neonatal Network"13, a mortalidade para o escore de CRIB >10 não atingiu 80\%, mostrando, assim, objetivo pelo qual devem ser desenvolvidas estratégias para redução do índice de $100 \%$, encontrado no presente estudo.

O escore de CRIB constitui um método de avaliação e comparação de populações, não apresentando capacidade de prognóstico acurado para ditar decisões individuais frente a um determinado paciente $^{6}$. O estudo original, realizado no Reuno Unido, merece ainda avaliações internacionais, com ênfase sobre possíveis influências raciais sobre o resultado.

O escore de CRIB constitui forma simples e objetiva de caracterizar a gravidade dos pacientes admitidos em UTI neonatal. A análise da mortalidade segundo o escore permite identificar o grupo populacional de maior risco para morte, além de se tornar num instrumento padronizado para comparação das taxas de mortalidade entre diferentes serviços. Poderão ser implementados programas com o objetivo de melhorar a qualidade assistencial, e o desempenho final da instituição poderá ser satisfatoriamente demonstrado pela aplicação do mesmo escore. 


\section{REFERÊNCIAS}

1. FLETCHER, R. H. Epidemiologia clínica. São Paulo, Artes Médicas, 1989.

2. GORTMAKER, S. et al. The survival of very low birthweight infants by level of hospital of birth: a population study of perinatal systems in for states. Am. J. Obstet. Gynecol., 152:517-24, 1985.

3. GREENLAND, S. et al. The case control method in medical care evaluation. Med. Care, 29:872-88, 1981.

4. HACK, M. et al. Very low birthweight outcomes of the National Institute of Child Health and Human Development Neonatal Network. Pediatrics, 87:587-97, 1991.

5. HANLEY, J. A. et al. A method of comparing the areas under the receiver operating characteristic curves derived from the same cases. Radiology, 148:839-43, 1983.

6. HOPE, P. CRIB, son of Apgar, brother to APACHE. Arch. Dis. Child., 72:F81-F83, 1995.

7. INVESTIGATORS OF THE VERMONT-OXFORD TRIALS NETWORK DATABASE PROJECT. The VermontOxford Trials Network: very low birth weight outcomes for 1990. Pediatrics, 91:540-5, 1993.
8. KNAUS, W.A. et al. APACHE II a severety of disease classification system. Crit. Care Med., 13:818-28, 1985.

9. PANETH, N. et al. Newborn intensive care and neonatal mortality in low birthweight infants: a population study. N. Engl. J. Med., 307:149-55, 1982.

10. POLLACK, M. M. et al. Pediatric risk of mortality (PRISM) score. Crit. Care Med., 16: 1110-6, 1988.

11. RICHARDSON, D. et al. Score for neonatal acute physiology (SNAP): a physiology based severity of illness index. Pediatr. Res., 29:262A, 1991

12. RICHARDSON, D. K. et al. Birthweight and illness severity: independent predictors of NICU mortality. Pediatrics, 91:969-75, 1993.

13. TARNOW-MORDI, W. et al. The CRIB (clinical risk index for babies) score: a tool for assessing initial neonatal risk and comparing performance of neonatal intensive care units. Lancet, 1: 193-8, 1993.

14. WILLIANS, R. L. Measurement the effectiveness of perinatal care. Med. Care, 17:95-100, 1979. 